\title{
Variables affecting mediation in children's verbal-motor paired-associate learning
}

\author{
JOAN H. CANTOR and WHEI-WEN SU \\ University of Iowa, Iowa City, Iowa 52242
}

\begin{abstract}
Fourth-grade children learned both names and motor responses for random shapes in a paired-associate learning task. Two levels of name similarity and two levels of stimulus similarity were combined in a 2 by 2 factorial design. The hypothesized development of mediated verbal-motor associations was assessed in terms of consistency in verbal-motor responding. Significant evidence of mediation was obtained in all experimental groups, and the relative strength of the mediational effect among groups was as follows: DD (dissimilar stimuli, dissimilar names) $=$ SD (similar stimuli, dissimilar names) $>$ SS (similar stimuli, similar names) > DS (dissimilar stimuli, similar names). The results were discussed in terms of the possible effects of the variables on the tendency to adopt a rehearsal strategy.
\end{abstract}

Verbal mediation has long been assigned an important role by theorists in explaining children's performance in a wide variety of situations including stimulus generalization, discrimination learning, verbal learning, and memory tasks. The postulation of mediational mechanisms, including verbal mediation, has been particularly prominent in the field of discrimination learning (Kendler \& Kendler, 1968; Spiker, 1970, 1971). Furthermore, there is now considerable evidence that verbal mediation plays an increasingly important role with age (e.g., Kendler, 1972; McGeehan \& Cantor, 1977), and that young children frequently show evidence of "mediational deficiency" (Reese, 1962). One of the two hypothesized contributors to the young child's failure to mediate is referred to as "production deficiency" (Flavell, Beach, \& Chinsky, 1966), that is, the failure to produce the potential mediating response. The second contributor is "control deficiency" (Kendler, 1972), which is the failure of the mediating response, when it does occur, to mediate or control other behavior. Thus, it is postulated, within an S-R framework, that two associations underlie the occurrence of a verbally mediated response: one between the external stimulus and the verbal response $\left(S \rightarrow R_{V}\right)$, and a second between the verbal response-produced stimulus and the instrumental response of interest $\left(s_{V} \rightarrow R_{I}\right)$.

This paper is based on a thesis submitted by the second author in partial fulfillment of the requirements for the MA degree at the University of Iowa. The first author supervised the thesis and is primarily responsible for preparation of the manuscript. The authors wish to thank Charles C. Spiker for a very helpful reading of the manuscript. Sincere appreciation is expressed to David L. Cronin, Superintendent, and many other personnel of the Iowa City Community School District for their fine cooperation in making subjects available. Thanks are also due to the Graduate College of the University of Iowa for funds to purchase computer time. Requests for reprints should be sent to Joan H. Cantor, Department of Psychology, University of Iowa, Iowa City, Iowa 52242.
Children may fail to show evidence of mediation, then, because either or both of these associations fail to develop.

Cantor $(1970,1973)$ has studied the concurrent development of both of these hypothesized associations in a combined verbal and motor task. In these studies, older children learned nonsense-syllable names and button-pushing responses concurrently for a set of three similar random shapes. On each trial, the subject was required to say the name aloud before he pushed the button. Evidence of mediation was provided by consistency in verbal and motor responding. More specifically, there was a strong tendency for the verbal and motor responses to be either both correct or both incorrect. If the subject made a correct verbal response, there was a high probability that he would make a correct motor response. If he made an incorrect verbal response, there was a high probability that he would make a "consistent" motor error, that is, make the motor response that was appropriate for the verbal response he had given. Subsequently, McGeehan and Cantor (1977) adapted this method for use with younger children ranging in age from 5 to 9 years. There was again a strong tendency for the verbal and motor responses to be learned in consistent pairs at all age levels, and, as expected, this tendency increased with age.

The analysis of verbal-motor consistency in this situation appears to provide a highly sensitive method of studying the factors affecting mediation in children. Although there is clear evidence of both production and control deficiency in younger children (Kendler, 1972; McGeehan \& Cantor, 1977), relatively little is known about the stimulus and response variables that affect the tendency to mediate. In the present study, the combined verbal-motor task was used to study the effects of stimulus similarity and verbal-response (name) similarity on the performance of fourth-grade children. 
Two levels of name similarity and two levels of stimulus similarity were used in factorial combination. On each trial, one member of a set of four random shapes was presented above an array of four identical wooden blocks. The child's task was first to give the correct name of the figure (shape) presented, and then to choose the correct block (spatial position) for that figure. The specific interest was in the effects of the similarity variables on nediation. Evidence for mediation is provided by conditional probabilities, which will be defined following a description of the theoretical analysis.

The S-R associations that are assumed to develop in the present learning task are diagrammed on the left side of Figure 1. The requirements of the task are assumed to result in the development of figure-name associations (e.g., $S_{1} \rightarrow R_{V_{1}}$ ), figure-block associations (e.g., $S_{1} \rightarrow R_{M_{1}}$ ), and name-block (mediated) associations (e.g., $\mathrm{SV}_{1} \rightarrow \mathrm{R}_{\mathrm{M}_{1}}$ ). Motor performance on a particular trial is assumed to be a function of the strength of both the figure-block and name-block associations. As noted earlier, consistency in verbalinotor performance is the major source of evidence for mediation. A motor response is consistent if it follows the particular verbal response that is paired with it in the learning task. For example, $\mathbf{R}_{\mathbf{M}_{1}}$ is consistent when it follows $R_{V_{1}}$. The generalized representation of consistent motor responses on the right side of Figure 1 shows that they may be either correct responses or errors. On a correct naming trial $\left(R_{V_{j}}\right.$ is made to $\left.S_{j}\right)$, the mediated association $\left(s_{V_{j}} \rightarrow R_{M_{j}}\right)$ does not compete with the correct figure-block association $\left(S_{j} \rightarrow R_{M_{j}}\right)$; rather, the two associations combine to produce a stronger tendency for the correct motor response to occur. Thus, a correct motor response following a correct verbal response is always consistent. On an incorrect naming trial $\left(R_{V_{k}}\right.$ is made to $\left.S_{j}\right)$, the mediated association $\left(\mathrm{s}_{\mathbf{k}} \rightarrow \mathrm{R}_{\mathbf{M}_{\mathbf{k}}}\right)$ may be sufficiently

\section{CORRECT ASSOCIATIONS IN VERBAL-MOTOR TASK}
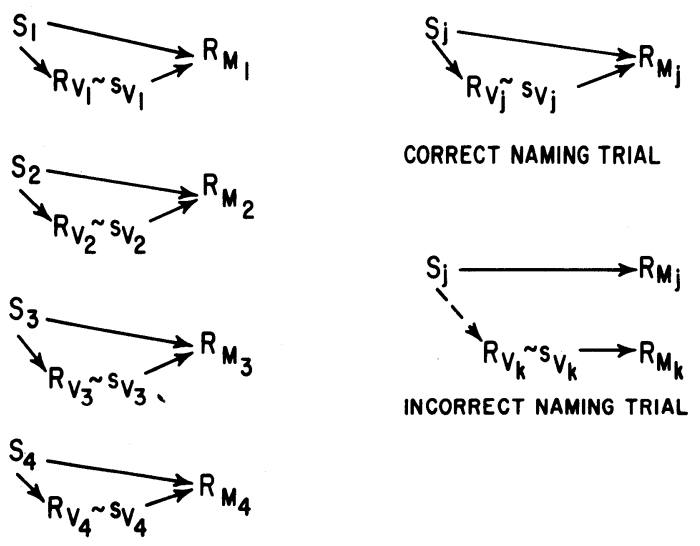

Figure 1. A theoretical analysis of associations learned in the verbal-motor task. strong to successfully compete with the correct figureblock association $\left(S_{j} \rightarrow R_{M_{j}}\right)$, thereby producing a consistent error $\left(R_{M_{k}}\right)$. An example of a consistent error would be the occurrence of $R_{M_{2}}$ following $R_{V_{2}}$ when $S_{1}$ is presented. Nonconsistent motor responses may also be either correct responses or errors.

On the basis of the above analysis, two predictions can be made regarding the motor performance of mediating subjects. (1) The probability of a correct motor response should be greater on correct-naming trials than on incorrect-naming trials [i.e., $\mathrm{P}(\mathrm{M} \mid \mathrm{V})>\mathrm{P}(\mathrm{M} \mid \overline{\mathrm{V}})$. Mediated correct responses should increase the first probability and mediated errors should decrease the second probability. In the absence of any mediation, motor responding should be independent of verbal responding, and the two probabilities should be equal. (2) On incorrect-naming trials, the probability of a consistent motor error should be greater than an adjusted probability of a nonconsistent error [i.e., $\mathrm{P}\left(\overline{\mathrm{M}}_{\mathrm{cs}} \mid \overline{\mathrm{V}}\right)>\mathrm{P}\left(\overline{\mathrm{M}}_{\text {ncs }} \mid \overline{\mathrm{V}}\right) / 2$ ]. In the latter measure, the probability of nonconsistent errors is divided by two, since there are two ways in which a nonconsistent error can occur, and only one way in which a consistent error can occur. Mediated errors should increase the first probability and decrease the second probability. In the absence of any mediation, the two probabilities should be equal.

\section{METHOD}

\section{Design and Subjects}

The use of two levels of stimulus similarity and two levels of name similarity in factorial combination resulted in four groups designated as DD, DS, SD, and SS, where the first letter represents dissimilar or similar stimuli, and the second letter represents dissimilar or similar names. A total of 144 fourthgrade children in the Iowa City Community School District served as subjects. Thirty-six children were randomly assigned to each of the four groups. The children ranged in age from 9 years 0 months to 10 years 0 months, with a mean age of 9 years 9 months.

\section{Stimuli and Apparatus}

Two sets of four random shapes were used as stimuli. The four similar stimuli were selected from sets of random shapes developed by Somnapan (1961), and the dissimilar stimuli were selected from the 24-point random shapes developed by Vanderplas and Garvin (1959). The specific figures used have been reproduced elsewhere (Su, 1975). Each figure was photographed and appeared as a black figure centered in a $5 \times 5 \mathrm{~cm}$ white square.

Eight nonsense syllables were used as stimulus names. The similar names were GEV, VEK, KEZ, and ZEG, and the dissimilar names were BIJ, VEK, DAX, and ZOL. The names were well matched with respect to meaningfulness values (Archer, 1960). Each name was printed in the center of a white cardboard square, $5 \mathrm{~cm}$ on a side.

Four black, round, wooden blocks were used in the motor task. They were arranged in an arc on the platform of a $55.9 \times 45.7 \mathrm{~cm}$ rectangular gray turntable. The distance between the centers of adjacent blocks was $13.3 \mathrm{~cm}$. Each block was $7.6 \mathrm{~cm}$ in diameter and was hinged on the back. Opening the block revealed a marble well that was used to bait the correct block on each trial. The turntable was rotated between trials, 
and a $45.7 \times 35.6 \mathrm{~cm}$ vertical screen located along the midline of the turntable prevented the subject from observing as the experimenter prepared the apparatus for the next trial. A small platform mounted above the blocks in the center of the vertical screen at approximately eye level was used to present the stimulus on each trial.

\section{Procedure}

Each child was tested individually in a mobile research trailer. The child was first given some practice trials on a simplified verbal-motor task. A circle and a square, which served as stimuli, were named "Pete" and "George," and each was associated with one of two blocks used for the motor responses. The child was told that his task was to first give the name of the figure and then to open the block that went with that figure. He was further told that if he opened the correct block, he would find a marble. Pretraining continued until the child made four consecutive correct responses.

Following pretraining, the child was shown each of the four stimuli for his similarity condition for approximately $5 \mathrm{sec}$ and was told to try to remember what each one looked like. Children in all groups were then given name familiarization in order to reduce the number of extralist verbal errors.

Verbal and motor training began immediately after the name familiarization. The experimenter gave the name of each stimulus and pointed to the correct block, allowing approximately $2.5 \mathrm{sec}$ of study time. All children were given 40 trials (stimulus presentations), with every stimulus presented twice in each block of eight trials. The anticipation interval was not strictly controlled, but the child was urged to respond after approximately $4 \mathrm{sec}$. During an interval of approximately $2.5 \mathrm{sec}$ following the child's motor response, the experimenter repeated the correct name and pointed to the correct block for the stimulus being displayed.

\section{Response Measures}

Only those verbal-motor trials in which the child responded with one of the four designated names were considered in analyzing the data, since evidence pertaining to mediation could not be obtained from trials in which the child used other names. The basic response measures used to compute the conditional probabilities were the following: frequencies of correct verbal responses (V), correct motor responses (M), trials in which verbal and motor responses were both correct (VM), trials in which verbal and motor responses were both incorrect $(\overline{\mathrm{VM}})$, and trials in which the verbal response was incorrect and the motor response was correct $(\overline{\mathrm{V}} \mathrm{M})$. The $\overline{\mathrm{V}} \overline{\mathrm{M}}$ trials were further divided into two categories: consistent errors $\left(\overline{\mathrm{M}}_{\mathrm{cs}}\right)$ and nonconsistent errors $\left(\overline{\mathrm{M}}_{\mathrm{ncs}}\right)$. The frequencies were converted into proportions, and the conditional probabilities were computed in the conventional manner [e.g., $\mathbf{P}(\mathrm{M} \mid \mathrm{V})=\mathrm{VM} / \mathrm{V}]$.

\section{RESULTS}

\section{Overall Performance}

The proportions of correct verbal and correct motor responses for the four groups were computed for each block of eight trials. Learning occurred in all groups, and both stimulus-similarity and name-similarity effects were evident, with Group DD showing the best performance and Group SS showing the poorest performance. The mean proportions of correct verbal responses combined over trials for Groups DD, DS, SD, and SS were $.70, .52, .57$, and .46 , respectively. The mean proportions of correct motor responses for these four groups were $.72, .58, .57$, and .50, respectively. An analysis of variance was performed for each measure separately, with trial blocks as a within-subjects factor, and name similarity and stimulus similarity as between-subjects factors. In the analysis of correct verbal responses, significant effects were obtained for trial blocks, name similarity, and stimulus similarity $[F(4,560)=111.56, p<.001 ; \quad F(1,140)=$ $24.71, \mathrm{p}<.001 ;$ and $\mathrm{F}(1,140)=11.62, \mathrm{p}<.001$, respectively]. The same results were obtained for correct motor responses $[F(4,560)=103.37, p<.001$; $\mathrm{F}(1,140)=13.68, \quad \mathrm{p}<.001 ;$ and $\mathrm{F}(1,140)=16.39$, $\mathrm{p}<.001$, respectively]. No significant interactions were obtained in either analysis.

\section{Verbal-Motor Performance}

The four conditional probabilities of interest were derived for each trial block on an individual-subject basis, as described earlier. Members of one pair of measures, $\mathrm{P}(\mathrm{M} \mid \mathrm{V})$ and $\mathrm{P}(\mathrm{M} \mid \overline{\mathrm{V}})$, were compared to test for mediational effects on correct motor responses. Members of the second pair, $\mathrm{P}\left(\overline{\mathrm{M}}_{\mathrm{cs}} \mid \overline{\mathrm{V}}\right)$ and $\mathrm{P}\left(\overline{\mathrm{M}}_{\mathrm{ncs}} \mid \overline{\mathrm{V}}\right) / 2$, were compared to test for mediational effects on motor errors. A four-way analysis of variance was performed for each pair of measures, with trial blocks and measures as within-subjects factors, and with name similarity and stimulus similarity as between-subjects factors. If the mediational hypothesis is correct, there should be a significant measure effect in each case. Interactions between measures and either or both of the similarity variables (name and stimulus) are of particular theoretical interest. In reporting the significant effects relevant to each major prediction, only the main effects and those interactions involving measures will be described.

(1) The probability of correct motor responses should be greater on correct naming trials than on incorrect naming trials [i.e., $\mathrm{P}(\mathrm{M} \mid \mathrm{V})>\mathrm{P}(\mathrm{M} \mid \overline{\mathrm{V}})$ ]. The group means combined over trial blocks are presented in the top half of Table 1 . It can be seen that $P(M \mid V)$ is markedly higher than $\mathrm{P}(\mathrm{M} \mid \overline{\mathrm{V}})$ in all four groups. Also, it can be seen that the difference appears to be smallest in Group DS, moderately large in Group SS, and largest in Groups DD and SD, which do not appear to differ from each other. The overall analysis showed significant main effects of trial blocks and measures

Table 1

Means of Conditional Probabilities for Each Experimental Group

\begin{tabular}{lcccc}
\hline & \multicolumn{5}{c}{ Group } \\
\cline { 2 - 5 } & DD & DS & SD & SS \\
\hline P(M $\mid \bar{V})$ & .91 & .75 & .87 & .78 \\
P(M $\mid \bar{V})$ & .14 & .33 & .10 & .17 \\
Difference & .77 & .42 & .77 & .61 \\
P(M $\left.\overline{\mathbf{M}}_{\text {cs }} \mid \overline{\mathbf{V}}\right)$ & .77 & .45 & .81 & .68 \\
P(M) & .04 & .11 & .05 & .07 \\
Difference & .73 & .34 & .76 & .61 \\
\hline
\end{tabular}


$[\mathrm{F}(4,560)=15.42, \mathrm{p}<.001 ;$ and $\mathrm{F}(1,140)=1,023.76$, $\mathrm{p}<.001$, respectively]. Significant interactions were obtained for Measures by Trial Blocks $[\mathrm{F}(4,560)=31.58$, $\mathrm{p}<.001]$, Measures by Name Similarity $[\mathrm{F}(1,140)=$ $40.38, \mathrm{p}<.001]$, Measures by Stimulus Similarity $[F(1,140)=5.70, p=.018]$, and Measures by Name Similarity by Stimulus Similarity $[F(1,140)=5.88$, $\mathrm{p}=.017]$. Follow-up tests of these interactions indicated that all groups showed a significant mediational effect, and that the relative strength of the effect among groups was the following: $\mathrm{DD}=\mathrm{SD}>\mathrm{SS}>\mathrm{DS}$.

(2) The probability of consistent motor errors on incorrect naming trials should be greater than the adjusted probability of nonconsistent errors [i.e., $\left.\mathrm{P}\left(\overline{\mathrm{M}}_{\mathrm{cs}} \mid \overline{\mathrm{V}}\right)>\mathrm{P}\left(\overline{\mathrm{M}}_{\text {ncs }} \mid \overline{\mathrm{V}}\right) / 2\right]$. The group means for these measures are presented in the bottom half of Table 1. Again, there are large differences in all groups, and the pattern of differences is the same as that found for correct responses. The overall analysis showed significant main effects of trial blocks and measures $[\mathrm{F}(4,560)=$ $10.55, \mathrm{p}<.001 ;$ and $\mathrm{F}(1,140)=998.57, \mathrm{p}<.001$, respectively]. Significant interactions were obtained for Measures by Trial Blocks $[\mathrm{F}(4,560)=27.31, \mathrm{p}<.001]$, Measures by Name Similarity $[F(1,140)=48.81$, $\mathrm{p}<.001]$, Measures by Stimulus Similarity $[\mathrm{F}(1,140)=$ $15.20, \mathrm{p}<.001]$, and Measures by Name Similarity by Stimulus Similarity $[F(1,140)=9.18, p=.003]$. Followup tests of the interactions indicated the same pattern of results found for correct responses.

\section{DISCUSSION}

Strong evidence of mediation was obtained in all groups. These results are consistent with earlier findings for conditions in which similar stimuli were used. Cantor $(1970,1973)$ used conditions similar to Groups SD and SS with older children and found evidence of mediation in both conditions. McGeehan and Cantor (1977) also found strong mediational effects for fourth-graders in a condition similar to Group SD.

Of particular interest here are the effects of stimulus and name similarity on the tendency to mediate. Cantor (1970, Experiment 2) had previously found greater evidence of mediation with dissimilar names than with similar names, a result that could be explained on the basis of generalization effects. Although the present results confirm the earlier findings, they also point to a rather surprising interaction between stimulus and name similarity. It will be recalled that the relative strengths of mediation among the groups were the following: $\mathrm{DD}=\mathrm{SD}>\mathrm{SS}>\mathrm{DS}$. Contrary to what might be expected on the basis of generalization effects, mediation was not reduced by increased stimulus similarity when the names were dissimilar $(\mathrm{DD}=\mathrm{SD})$. Furthermore, mediation was actually greater for similar stimuli than for dissimilar stimuli when the names were similar (SS > DS).

Our hypothesis is that these results reflect differential strategies used by children in the various similarity conditions. Spiker (1956) suggested that subjects may adopt the strategy of using verbal labels for stimuli to rehearse the correct motor responses between trials. More recently, investigators (e.g., Flavell et al., 1966; McGeehan \& Cantor, 1977) have suggested that failure to rehearse may be responsible for control deficiency in young children. In the present task, rehearsal would take the form, "VEK goes with Block 2," thus theoretically strengthening the mediated name-button associations.

If we are correct that the similarity variables affect the tendency to rehearse, then the results point to two conclusions. First, the children have a strong tendency to rehearse with distinctive names under all conditions of stimulus similarity $(D D=S D)$, since it is very easy to do so. Second, the children are less likely to adopt a rehearsal strategy with similar names, since it is more difficult to do so, and this tendency is reduced even further when the stimuli are distinctive (SS $>$ DS). In other words, only when the stimuli are hard to discriminate do the children resort to the relatively difficult task of rehearsing with similar names (Group SS). In Group DS, on the other hand, it is perhaps easier to associate the correct block directly with each (distinctive) stimulus. Although these assumptions about how situational variables affect mediation through a rehearsal strategy account well for the present results, additional research will of course be needed to verify the assumptions and to determine their usefulness in explaining other mediational effects in children.

\section{REFERENCES}

ARCHER, E. J. Re-evaluation of the meaningfulness of all possible CVC trigrams. Psychological Monographs, 1960, 74, 1-23.

Cantor, J. H. Facilitating and interfering effects of stimulus naming on children's motor paired-associate learning. Journal of Experimental Child Psychology, 1970, 10, 374-389.

Cantor, J. H. Stimulus naming effects at different stages of motor paired-associate learning. Memory \& Cognition, 1973, 1, 47-52.

Flavell, J. H., Beach, D. R., \& Chinsky, J. M. Spontaneous verbal rehearsal in a memory task as a function of age. Child Development, 1966, 37, 283-299.

Kendler, H. H., \& Kendler, T. S. Mediation and conceptual behavior. In J. T. Spence (Ed.), The psychology of learning and motivation (Vol. 2). New York: Academic Press, 1968.

KENDLER, T. S. An ontogeny of mediational deficiency. Child Development, 1972, 43, 1-17.

McGeehan, D. L., \& Cantor, J. H. Age differences in mediation in children's motor paired-associate learning. Developmental Psychology, 1977, 13, 121-128.

REESE, H. W. Verbal mediation as a function of age level. Psychological Bulletin, 1962, 59, 502-509.

SomnAPAN, R. Relative discriminability of random shapes. Unpublished master's thesis, University of Iowa, 1961.

SPIKER, C. C. Stimulus pretraining and subsequent performance in the delayed reaction experiment. Journal of Experimental Psychology, 1956, 52, 107-111.

SPIKER, C. C. An extension of Hull-Spence discrimination learning theory. Psychological Review, 1970, 77, 496-515.

SPIKER, C. C. Application of Hull-Spence theory to the discrimination learning of children. In H. W. Reese (Ed.), Advances in child development and behavior (Vol. 6). New York: Academic Press, 1971.

$\mathrm{Su}, \mathrm{W}$. W. The effects of name similarity and stimulus similarity on children's verbal-motor paired-associate learning. Unpublished master's thesis, University of Iowa, 1975.

Vanderplas, J. M., \& Garvin, E. A. Complexity, association value, and practice as factors in shape recognition following paired-associates training. Journal of Experimental Psychology, 1959, 57, 155-163.

(Received for publication September 6, 1978.) 